\title{
Simultaneous Detection and Differentiation of Chikungunya Virus and Dengue Virus in Blood at Clinical Presentation by Real Time RT-PCR
}

Rahman MM', Rahim $\mathrm{R}^{2}$, Rasel $\mathrm{AH}^{3}$, Murad $\mathrm{AS}^{4}$

\begin{abstract}
Background: Chikungunya viral infection in Bangladesh has marked 2017 summer with unprecedented magnitude for the first time. Chikungunya virus (CHIKV) and Dengue virus (DENV) are arboviruses that share the same Aedes mosquito vectors and thus overlap in their endemic areas. These two viruses cause similar clinical presentations, especially in the initial stages of infection. Because the outcomes and management strategies for these two viruses are different, early and accurate diagnosis is imperative. Early diagnosis is also important for surveillance and outbreak control. Objective: As virus isolation is not undergoing in the country and antigen based commercial detection assay is not available for CHIKV we used one step real time reverse transcriptase polymerase chain reaction (RT-PCR) method to detect and discriminate CHIKV and DENV in blood during suspicious clinical symptoms. Results: By this RT-PCR method we have detected 603 cases of CHIKV and 233 cases of DENV and thus facilitated rapid diagnosis and clinical management in the recent CHIKV outbreaks in the country. Conclusion: This is the first report about molecular detection and differentiation of CHIKV and DENV at time of clinical presentation and further show evidence of simultaneous outbreaks of both the viral infections in the country.
\end{abstract}

\section{Key Words}

Arbovirus, Chikungunya virus, Dengue virus, RT PCR

\section{Introduction}

Chikungunya virus (CHIKV) and Dengue virus (DENV) are single - stranded, positive - sense RNA viruses. DENV belongs to the family Flaviviridae and genus Flavivurus and CHIKV belongs to the family Togaviridae and genus Alphavirus of which there are 3 known strains
(West African; East-Central-South African, Asian). ${ }^{1}$ The genome of each virus is approximately $11 \mathrm{~kb}$ in length. ${ }^{1,2}$ The CHIKV genome encodes three structural (C, E1, and E2) and four nonstructural (nsP1-4) proteins. ${ }^{1}$ The DENV genome encodes three structural (C, prM, and E) and seven nonstructural (NS1, NS2B, NS3,

1. Sr. Consultant, Molecular Diagnostics, Apollo Hospitals Dhaka 2. Sr. Registrar, Molecular Diagnostics, Apollo Hospitals Dhaka 3. Scientific Officer, Molecular Diagnostics, Apollo Hospitals Dhaka 4. Sr. Medical Technologist, Molecular Diagnostics, Apollo Hospitals Dhaka 
NS3, NS4A, NS4B, and NS5) proteins. ${ }^{3}$ Both viruses are arthropod-borne viruses (arboviruses) sharing a common vector: mosquitos of the Aedes genus, specifically A. aegypti and A. albopictus. ${ }^{4}$ Both viruses circulate in similar geographic regions. Concurrent infection with both viruses, transmitted from either two different mosquitos or one dually infected mosquito, is possible.5,6 For DENV, transmission has also been reported to occur via infected blood products, organ donation, and prenatal and/or perinatal vertical transmission. ${ }^{7}$

While CHIKV and DENV present similarly as an acute febrile illness, these two viruses have different management strategies and outcomes. The majority of CHIKV infections are selflimiting with chronic joint disease being the most common long - term outcome and fatality is exceedingly rare. Nonsteroidal anti- inflammatory drugs (NSAIDs) are the mainstay treatment for CHIKV but NSAIDs should be avoided until DENV is confidently ruled out, as NSAIDs are contraindicated in DENV infection. ${ }^{8}$ DENV is likewise commonly a self-limiting illness, yet this diagnosis necessitates stricter monitoring due to the potential for more significant morbidity and mortality.

In Bangladesh, Dengue fever (DF) was documented from mid-1960s to mid-1990s and Dengue hemorrhagic fever (DHF) from 2000.9 CHIKV was first isolated from a febrile individual in Tanzania in 1952. Since then, CHIKV has caused numerous well-documented outbreaks and epidemics in both Africa and Southeast Asia, involving hundreds of thousands of people. ${ }^{10}$ In Bangladesh, CHIKV infection was serologically first confirmed in 2008.11 Thereafter, infrequent outbreaks and only a few cases of chikungunya were reported from Bangladesh. ${ }^{22,13}$ This year it has emerged as an important public health issue. ${ }^{14}$ Despite the fact that CHIKV resurgence is associated with epidemics of unprecedented magnitude, only a few serologic studies in this country have found CHIKV-specific antibodies in patients who were suspected of having dengue virus infection, but no reports have been published on the molecular detection of CHIKV at time of clinical presentation. Because infection with CHIKV and DENV should be on the differential diagnosis together at the initial patient presentation, tests that screen for these viruses simultaneously are preferred to test for them separately. CHIKV and DENV are not readily differentiated serologically due to cross-reactivity of their serocomplexes, so there is a reliance on molecular detection methods for this purpose. 15 A one-step duplex conventional RT-PCR assay for distinguishing CHIKV and DENV has been reported. ${ }^{16}$ We used commercial one step real time RT-PCR kit for detecting and distinguishing CHIKV and DENV in blood at clinical presentation of clinically suspected patients. 


\section{Materials and Methods}

\section{Patients and clinical specimens}

$3 \mathrm{ml}$ whole blood sample from adult and $0.5 \mathrm{ml}$ to $1 \mathrm{ml}$ from children having clinical suspicion of either chikungunya or dengue were collected in plain vacutainer (red top) of patients who visited Apollo Hospitals Dhaka, Bangladesh during June 29, 2017 to October 31, 2017. Samples were centrifuged within hours and serum were stocked at $-80^{\circ} \mathrm{C}$ until doing extraction of RNA.Viral RNA was extracted from 200 ul of serum following kit manufacturer's protocol (QIAampMinElute Virus Spin Kit, Qiagen, Germany) and stored at $-80^{\circ} \mathrm{C}$ if not used immediately.

\section{Diagnostic RT-PCR}

We used commercial one step reverse transcriptase real time PCR kit from FTD (Fast Track Diagnostics), Luxembourg for the detection and differentiation of chikungunya virus and dengue virus. FTD Dengue/Chik is a CE-IVD approved in vitro test for the detection of viral nucleic acid in whole blood and urine as an aid in the evaluation of dengue fever caused by dengue virus and fever diseases caused by chikungunya virus. To control the extraction and to exclude PCR inhibition, the test includes Brome mosaic virus (BMV) as an internal control. FTD Dengue/Chik contains the primer and probe mix (PP), the positive control (PC), the negative control (NC) and an internal control (IC). The kit specificity was validated with various negative samples tested by a reference method. The negative samples did not generate any positive signals with FTD Dengue/Chik (FTD 43 - 32_64 - VALIDATION - v2 2016_12 EN).

\section{Principle of the method}

The viral RNA is transcribed into cDNA using a specific primer mediated reverse transcription step followed immediately in the same tube by polymerase chain reaction. The presence of specific pathogen sequences in the reaction is detected by an increase in fluorescence observed from the relevant dual-labeled probe, and is reported as a cycle threshold value $(\mathrm{Ct})$ by the Real-time thermocycler. The assay uses brome mosaic virus (BMV) as an extraction control- the internal control (IC)- which is introduced into each sample and the negative control at the lysis buffer stage of the extraction process.

\section{Main PCR setup procedure}

15ul PCR master mix containing 12.5 ul buffer, 1.5ul PPmix and 1ul enzyme is prepared in 1.5 $\mathrm{ml}$ microcentrifuge tube for each sample, negative control and positive control and then 10ul of the extracted RNA from samples, the extracted negative control and positive control is added, respectively. Then it is briefly mixed by up and transferred the amount in $0.1 \mathrm{ml}$ PCR tube and placed it on thermocycler, Rotor Gene Q. According to kit manufacturer's instruction thermocycler is programmed which is briefly as hold at $50^{\circ} \mathrm{C}$ for 15 minutes, again hold $1 \mathrm{~min}$ at $94^{\circ} \mathrm{C}$, then 40 cycles of 8 second at $94^{\circ} \mathrm{C}$ and 1 minute at $60^{\circ} \mathrm{C}$. 
Each run was performed with a negative control (no template) and positive controls for CHIKV and DENV. Signal was acquired at $60^{\circ} \mathrm{C}$, and analysis was performed on the linear scale. Thresholds were set manually on each run. For both targets, any exponential curve crossing this threshold was considered positive. Fluorescence curve detected in Orange Channel are for amplification of Chikungunya virus and fluorescence curve detected in Green Channel are for amplification of Dengue virus. Internal control is detected in red channel.

\section{Results and Discussion}

\section{Clinical features among suspected patients}

CHIKV and DENV, the two arboviruses share a similar geographic distribution and unfortunately, their clinical manifestations also reported substantial overlap. Patients came in the Apollo Hospitals Dhaka during June 29 to October 31 2017 with acute onset of fever, myalgia, arthralgia and headache and some patients experienced a maculopapular rash and/or gastrointestinal symptoms. In case of adults most dominating features along with fever was arthralgia with or without swelling. However, in case of children arthralgia was mild.

Table 1. Distribution of Suspected and Confirmed Cases of Chikungunya Virus and Dengue Virus

\begin{tabular}{|c|c|c|c|c|}
\hline Age & $\begin{array}{l}\text { Suspected cases } \\
\text { No. }\end{array}$ & CHIKV+, No. (X) & DenV+, No. (X) & $\begin{array}{l}\text { Co-infection, } \\
\text { No. }\end{array}$ \\
\hline 4 & 27 & $5(18.5)$ & $5(18.5)$ & 0 \\
\hline $1-10$ & 392 & $104(26.5)$ & $56(14.28)$ & 2 \\
\hline $11-20$ & 154 & $46(29.8)$ & $33(21.42)$ & 0 \\
\hline $21-30$ & 159 & $70(44)$ & 31 (19.49) & 2 \\
\hline $31-40$ & 202 & $101(50)$ & $38(18.80)$ & 1 \\
\hline $41-50$ & 181 & $74(40.8)$ & $40(22.09)$ & 2 \\
\hline $51 \cdot 60$ & 110 & $56(50.9)$ & $14(12.7)$ & 2 \\
\hline $61 \cdot 70$ & 137 & $83(60.5)$ & $10(7.29)$ & 1 \\
\hline$>70$ & 128 & $62(48.4)$ & $9(7.09)$ & 0 \\
\hline Total & 1500 & $601(40 \%)$ & $236(15.73)$ & 10 \\
\hline
\end{tabular}

Unfortunately, there is no single clinical sign available for distinguishing CHIKV and DENV infection from each other or from other acute febrile illnesses. Therefore, both of these viruses must be initially included in the differential diagnosis for a patient with suspicious clinical symptoms. Clinical features can serve, at best, as a guide for favoring one virus over the other, as patients may present atypically, either by lacking the "classic" signs or symptoms or by presenting in an uncharacteristic manner. Laboratory diagnostic tests are thus essential for accurate identification of the causative virus.

\section{Age distribution of suspected and confirmed cases of CHIKV and DENV infection}

Out of 1500 suspected cases 826 (55\%) were male and 674 (44.93\%) were female. It is noticeable that all age groups were infected by both the viruses, mostly separately (table 1). Confirmed CHIKV cases were found more in adult groups (49.1\%) than age groups below 20 years $(24.9 \%)$. However, no such dominance is seen in any age group among DENV positive cases. Among 1500 cases 601 (40\%) cases were found positive for CHIKV and 236 (15.73\%) cases were found positive for DENV and Co-infection was found in 10 cases.

\section{Detection and Differentiation of CHIKV and DENV by Real Time RT-PCR}

The acute febrile phase of infection corresponds to the period of viremia, which lasts typically 5 days after onset of fever for both CHIKV and DENV. During this time, diagnosis rests on isolation of the virus, viral RNA, or viral antigen 
from the specimen. As virus isolation is not undergoing in the country and antigen based commercial detection assay is not available for CHIKV we aimed to detect viral RNA. Further, compared to virus isolation, viral nucleic acid detection techniques performed on acute-phase specimens offer better sensitivity with a much more rapid turnaround time. Viral nucleic acid can be detected for a few additional days beyond the period of viremia.

We used commercial one step real time reverse transcriptase polymerase chain reaction (RTPCR) method to detect and discriminate CHIKV and DENV in blood with suspicious clinical symptoms at presentation during June

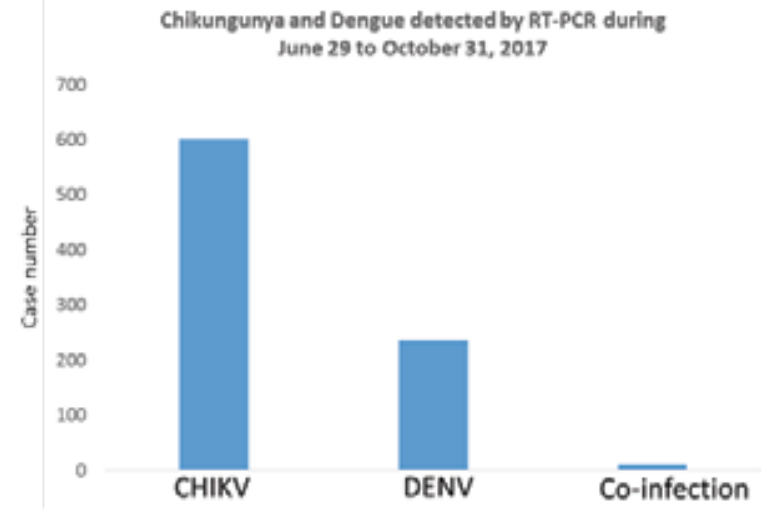

Fig. 1:Positive cases of Chikungunya virus and Dengue virus

29, 2017 to October 31, 2017. We tested serum from 1500 suspected patients and found 601positive cases of chikungunya virus and 236 positive cases of Dengue virus (Fig. 1). These findings show epidemic outbreaks of chikungunya virus infection in the country for the first time where dengue virus infection is also endemic for more than decades.

\section{Conclusion}

Confirmation of DENV or CHIKV infection requires laboratory diagnosis. The RT-PCR method we adopted to detect and differentiate CHIKV and DENV at time of clinical presentation clearly showed existence of both the arboviruses in the community with unprecedented magnitude of CHIKV for the first time in the country. As most patient seek medical services at time of clinical presentation only this RT-PCR method can detect the viral infections with certainty as viral load remains high at this period.

Molecular assays are more sensitive for diagnosis in the early stages of illness (1-4 days after onset) when antibodies are not detected. However, considering the cost and technical complexicity of RT-PCR method, an antigen based rapid detection system is imperative for wide spread detection of these viruses at time of clinical presentation.

\section{References}

1. Caglioti C, Lalle E, Castilletti C, Carletti F,Capobianchi MR, and Bordi L. Chikungunya virus infection: an overview. New Microbiologica,2013; 36(3):211-27.

2. SudeepAB and Parashar D. Chikungunya: an overview. Journal of Biosciences, 2008; 33(4): 443-9.

3. Tang KF and OoiEE. Diagnosis of dengue: an update. Expert Review of Anti-Infective Therapy, 2012; 10(8): 895-907.

4. Chen LH and WilsonME. Dengue and chikungunya infections in travelers. Current Opinion in Infectious Diseases, 2010; 23(5): 438-44.

5. Chen LHand WilsonME. Dengue and chikungunya in travelers: recent updates. Current Opinion in Infectious Diseases, 2012; 25(5):523-9.

6. LeeVJ, ChowA, Zheng X,Carrasco LR, Cook AR, Lye DC,et al. Simple clinical and laboratory predictors of Chikungunya versus dengue infections in adults.PLoS Neglected Tropical Diseases, 2012; 6(9): Article ID e1786.

7. HeilmanJM, WolffJD, BeardsGM, and BasdenBJ. Dengue fever: AWikipedia clinical review. OpenMedicine, 2014; 8(4): e105-e115. 
8.LaoprasopwattanaK,KaewjungwadL, JarumanokulR and GeaterA. Differential diagnosis of chikungunya, dengue viral infection and other acute febrile illnesses in children. Pediatric Infectious Disease Journal, 2012; 31(5): 459-63.

9. Rahman M, Rahman K, Siddique AK, Shoma S, KamaAHM,Ali KS, et al. First outbreak of Dengue Hemorrhagic fever, Bangladesh. Emerg Infect Dis, 2002; 8(7):73840 .

10. Schuffenecker I, Iteman I, Michault A, Murri S, Frangeul L, Vaney M-C, et al. Genome microevolution of chikungunya viruses causing the Indian Ocean outbreak. PLoS Med, 2006;3(7): e263.

11. First identified outbreak of Chikungunya in Bangladesh, 2008 Health and Science Bulletin, icddr,b, 2009; 7(1)

12. Hassan R, Rahman MM, Moniruzzaman M, Rahim A, Barua S, Biswas R, et al. Chikungunya-an emerging infection in Bangladesh: a case series. J Med Case Rep, 2014;8:67.
13. Khatun S, Chakraborty A, Rahman M, Banu N, Rahman MM, Hasan SMM, et al. An outbreak of chikungunya in rural Bangladesh, 2011. PLoSNegl Trop Dis, 2015;9(7): e0003907. 14. Hasib NI. Chikungunya viral disease spreads fast in Dhaka. http://bdnews24.com. Accessed 25 Jun 2017.

15. Saha K, Firdaus R, Chakrabarti S, and Sadhukhan PC. Development of rapid, sensitive one-tube duplex RT-PCR assay for specific and differential diagnosis of Chikungunya and dengue.Journal of Virological Methods, 2013; 193(2): $521-4$.

16. Dash PK, Parida M, Santhosh SR, Saxena P, Srivastava A, Neeraja M, et al.Development and evaluation of a 1-step duplex reverse transcription polymerase chain reaction for differential diagnosis of chikungunya and dengue infection. Diagnostic Microbiology and Infectious Disease, 2008; 62(1): $52-7$. 